PROCEEDINGS OF THE

AMERICAN MATHEMATICAL SOCIETY

Volume 134, Number 9, September 2006, Pages 2521-2531

S 0002-9939(06)08495-4

Article electronically published on March 22, 2006

\title{
SUPER SOLUTIONS OF THE DYNAMICAL YANG-BAXTER EQUATION
}

\author{
GIZEM KARAALI
}

(Communicated by Dan M. Barbasch)

\begin{abstract}
Solutions of the classical dynamical Yang-Baxter equation on a Lie superalgebra are called super dynamical $r$-matrices. A super dynamical $r$-matrix $r$ satisfies the zero weight condition if $[h \otimes 1+1 \otimes h, r(\lambda)]=0$ for all $h \in \mathfrak{h}, \lambda \in \mathfrak{h}^{*}$.
\end{abstract}

In this paper we classify super dynamical $r$-matrices with zero weight.

\section{INTRODUCTION}

A complete classification of the (constant) non-skewsymmetric solutions of the classical Yang-Baxter equation exists in the case when the underlying structure is a simple Lie algebra; see [1] and 2] for the original proofs by Belavin and Drinfeld, and [5] for a more pedagogical exposition. A similar construction, with natural modifications, works in the super case as well; see [8]. However, it turns out that this may not be easily modified into a full classification result; see 9$]$ for an explicit construction and detailed study of a counterexample.

It is well known that solutions of the classical Yang-Baxter equation on a Lie algebra give us the semiclassical limits of quantizations on the associated Lie group. In 4, Etingof, Schedler and Schiffmann explicitly constructed quantizations associated to all solutions coming from the Belavin-Drinfeld result. Their method in fact works for all dynamical $r$-matrices, i.e. the solutions of the more general classical dynamical Yang-Baxter equation.

The purpose of this paper is to initiate the study of the super analogue of the theory of dynamical $r$-matrices. In particular, we make the appropriate definitions and provide a full classification result for a class of super dynamical $r$-matrices. Ultimately our goal is a full theory of quantum groups in the super setting. We expect that understanding the super solutions of the dynamical Yang-Baxter equation will provide us with valuable insight, and hence help us extend or modify the quantization result cited above to obtain a graded analogue 1 Classification results

Received by the editors March 31, 2005.

2000 Mathematics Subject Classification. Primary 17B37.

Key words and phrases. Dynamical $r$-matrices, the dynamical Yang-Baxter equation, Lie superalgebras.

${ }^{1} \mathrm{~A}$ different method of quantization, by Etingof and Kazhdan, has been generalized to the super setting; see [7]. However, this is a less constructive technique and does not fully answer our questions.

(C)2006 American Mathematical Society Reverts to public domain 28 years from publication 
in this more general setting of dynamical $r$-matrices may also clarify the so far exceptional case of the classical $r$-matrices introduced in [9].

The two theorems proved in this paper are motivated by Theorem 3.2 and Theorem 3.10 in [6], and provide us with a full classification of super dynamical $r$-matrices with zero weight. For a classification of all super dynamical $r$-matrices, one needs further study. Schiffmann's results in [10] provide a good template. It is this author's belief that the constructive part of Schiffmann's results will carry over to the super case with certain modifications. In light of recent results, one expects to see some divergence from the non-graded theory when it comes to the classification part; in fact the example studied in [9] will be one of the challenges to a direct generalization.

\section{DYNAMICAL $r$-MATRICES IN THE SUPER SETTING}

2.1. Definitions. Let $\mathfrak{g}$ be a simple Lie superalgebra with non-degenerate Killing form $(\cdot, \cdot)$. Let $\mathfrak{h} \subset \mathfrak{g}$ be a Cartan subsuperalgebra, and let $\Delta \subset \mathfrak{h}^{*}$ be the set of roots associated to $\mathfrak{h}$. Fix a set of simple roots $\Gamma$ or equivalently a Borel $\mathfrak{b}$. The classical dynamical Yang-Baxter equation for a meromorphic function $r: \mathfrak{h}^{*} \rightarrow \mathfrak{g} \otimes \mathfrak{g}$ will be

$$
\operatorname{Alt}_{s}(d r)+[[r, r]]=0
$$

where, for any 2 -tensor $r$,

$$
[[r, r]]=\left[r^{12}, r^{13}\right]+\left[r^{12}, r^{23}\right]+\left[r^{13}, r^{23}\right] .
$$

The differential of $r$ will be defined as

$$
\begin{aligned}
& d r \quad: \quad \mathfrak{h}^{*} \quad \longrightarrow \quad \mathfrak{g} \otimes \mathfrak{g} \otimes \mathfrak{g} \\
& \lambda \longmapsto \sum_{i} x_{i} \otimes \frac{\partial r}{\partial x_{i}}(\lambda) .
\end{aligned}
$$

Here $\left\{x_{i}\right\}$ is a basis for $\mathfrak{h}$, so all the $x_{i}$ are even. Recall that $\operatorname{Alt}_{s}: \mathfrak{g}^{\otimes 3} \rightarrow \mathfrak{g}^{\otimes 3}$ is given on homogeneous elements by

$$
\operatorname{Alt}_{s}(a \otimes b \otimes c)=a \otimes b \otimes c+(-1)^{|a|(|b|+|c|)} b \otimes c \otimes a+(-1)^{|c|(|a|+|b|)} c \otimes a \otimes b .
$$

In view of all this, we can see that for $r=\sum_{i} R_{i(1)} \otimes R_{i(2)}, \operatorname{Alt}_{s}(d r)$ may be rewritten as

$$
\sum_{i} x_{i}^{(1)}\left(\frac{\partial r}{\partial x_{i}}\right)^{(23)}+\sum_{i} x_{i}^{(2)}\left(\frac{\partial r}{\partial x_{i}}\right)^{(31)}+\sum_{i}(-1)^{\left|R_{i(1)}\right|\left|R_{i(2)}\right|} x_{i}^{(3)}\left(\frac{\partial r}{\partial x_{i}}\right)^{(12)} .
$$

We will say that a meromorphic function $r: \mathfrak{h}^{*} \rightarrow \mathfrak{g} \otimes \mathfrak{g}$ is a super dynamical $r$-matrix with coupling constant $\epsilon$ if it is a solution to Equation (2.1) and satisfies the generalized unitarity condition

$$
r(\lambda)+T_{s}(r)(\lambda)=\epsilon \Omega
$$

where $\Omega$ is the Casimir element, i.e. the element of $\mathfrak{g} \otimes \mathfrak{g}$ corresponding to the Killing form. Here, $T_{s}: V \otimes V \rightarrow V \otimes V$ is the super twist map defined on the homogeneous elements of a given super vector space $V$ as

$$
T_{s}(a \otimes b)=(-1)^{|a||b|} b \otimes a .
$$

Remark 2.1. The above definitions are the natural super analogues of the nongraded terminology used in the study of the dynamical Yang-Baxter equation; see [3] for a survey on the non-graded theory. 
2.2. Super dynamical r-matrices with zero weight. A super dynamical $r$-matrix $r$ satisfies the zero weight condition if

$$
[h \otimes 1+1 \otimes h, r(\lambda)]=0 \text { for all } h \in \mathfrak{h}, \lambda \in \mathfrak{h}^{*} .
$$

In the next two sections we will prove the following two statements:

Theorem 2.2. Let $\mathfrak{g}$ be a simple Lie superalgebra with non-degenerate Killing form $(\cdot, \cdot), \mathfrak{h} \subset \mathfrak{g}$ a Cartan subsuperalgebra, and $\Delta \subset \mathfrak{h}^{*}$ the set of roots associated to $\mathfrak{h}$.

(1) Let $X$ be a subset of the set of roots $\Delta$ of $\mathfrak{g}$ such that:

(a) if $\alpha, \beta \in X$ and $\alpha+\beta$ is a root, then $\alpha+\beta \in X$, and

(b) if $\alpha \in X$, then $-\alpha \in X$.

Let $\nu \in \mathfrak{h}^{*}$, and let $D=\sum_{i<j} D_{i j} d x_{i} \wedge d x_{j}$ be a closed meromorphic 2 -form on $\mathfrak{h}^{*}$. If we set $D_{i j}=-D_{j i}$ for $i \geq j$, then the meromorphic function

$$
r(\lambda)=\sum_{i, j=1}^{N} D_{i j}(\lambda) x_{i} \otimes x_{j}+\sum_{\alpha \in X} \frac{(-1)^{|\alpha|}\left(e_{\alpha}, e_{-\alpha}\right)}{(\alpha, \lambda-\nu)} e_{\alpha} \otimes e_{-\alpha}
$$

is a super dynamical r-matrix with zero weight and zero coupling constant.

(2) Any super dynamical r-matrix with zero weight and zero coupling constant is of this form.

Theorem 2.3. Let $\mathfrak{g}$ be a simple Lie superalgebra with non-degenerate Killing form $(\cdot, \cdot), \mathfrak{h} \subset \mathfrak{g}$ a Cartan subsuperalgebra, and $\Delta \subset \mathfrak{h}^{*}$ the set of roots associated to $\mathfrak{h}$.

(1) Let $X$ be a subset of the set of roots $\Delta$ of $\mathfrak{g}$ satisfying conditions (a) and (b) of Theorem 2.2. Pick $\nu \in \mathfrak{h}^{*}$, and define

$$
\phi_{\alpha}= \begin{cases}(\epsilon / 2) \operatorname{coth}\left((-1)^{|\alpha|}\left(e_{\alpha}, e_{-\alpha}\right)(\epsilon / 2)(\alpha, \lambda-\nu)\right) & \text { if } \alpha \in X, \\ ( \pm \epsilon / 2) & \text { if } \alpha \notin X, \text { negative, } \\ \mp(-1)^{|\alpha|}(\epsilon / 2) & \text { if } \alpha \notin X, \text { positive. }\end{cases}
$$

Let $D=\sum_{i<j} D_{i j} d x_{i} \wedge d x_{j}$ be a closed meromorphic 2-form on $\mathfrak{h}^{*}$. If we set $D_{i j}=-D_{j i}$ for $i \geq j$, then the meromorphic function

$$
r(\lambda)=\sum_{i, j=1}^{N} D_{i j}(\lambda) x_{i} \otimes x_{j}+\frac{\epsilon}{2} \Omega+\sum_{\alpha \in \Delta} \phi_{\alpha} e_{\alpha} \otimes e_{-\alpha}
$$

is a super dynamical r-matrix with zero weight and non-zero coupling constant $\epsilon$.

(2) Any super dynamical r-matrix with zero weight and non-zero coupling constant $\epsilon$ is of this form.

Remark 2.4. Note that if $\epsilon \rightarrow 0$, Equation (2.4) reduces to Equation (2.3).

\section{Proof of Theorem 2.2}

Before we start the proof, we fix some notation: For any positive root $\alpha$ fix $e_{\alpha} \in \mathfrak{g}_{\alpha}$ and pick $e_{-\alpha} \in \mathfrak{g}_{-\alpha}$ dual to $e_{\alpha}$, i.e.

$$
\left(e_{\alpha}, e_{-\alpha}\right)=1 \text { for all } \alpha \in \Delta^{+} \text {. }
$$

Note that we can do this uniquely up to scalars because all the $\mathfrak{g}_{\alpha}$ are one dimensional (which follows from the non-degeneracy of the Killing form). Define

$$
A_{\alpha}=\left\{\begin{array}{cl}
(-1)^{|\alpha|} & \text { if } \alpha \text { is positive } \\
1 & \text { if } \alpha \text { is negative. }
\end{array}\right.
$$


Note that $A_{-\alpha}=(-1)^{|\alpha|} A_{\alpha}$. We can use $A_{\alpha}$ for instance to write the duals of our basis vectors in terms of one another:

$$
e_{\alpha}^{*}=A_{-\alpha} e_{-\alpha}
$$

or equivalently:

$$
\left(e_{\alpha}, e_{-\alpha}\right)=A_{-\alpha} .
$$

Proof. Let $r: \mathfrak{h}^{*} \rightarrow \mathfrak{g} \otimes \mathfrak{g}$ be a super dynamical $r$-matrix with zero coupling constant. Then the zero weight condition on $r$ implies that $r$ has to be of the form

$$
r(\lambda)=\sum_{i, j} D_{i j}(\lambda) x_{i} \otimes x_{j}+\sum_{\alpha \in \Delta} \phi_{\alpha} e_{\alpha} \otimes e_{-\alpha}
$$

where $D_{i j}, \phi_{\alpha}$ are suitable scalar meromorphic functions such that

$$
D_{i j}(\lambda)=-D_{j i}(\lambda) \quad \text { and } \quad \phi_{-\alpha}=-(-1)^{|\alpha|} \phi_{\alpha} .
$$

One can easily check that there can be no terms mixing the Cartan part with the non-Cartan part, and the conditions on the $D_{i j}$ and the $\phi_{\alpha}$ follow from the zero coupling constant.

Equation (2.1) is an equation in $\mathfrak{g} \otimes \mathfrak{g} \otimes \mathfrak{g}$. The zero coupling constant implies that the left-hand side of the equation is skew-symmetric with respect to signed permutations of factors. Therefore in order to solve Equation (2.1), it is enough to look at its $\mathfrak{h} \otimes \mathfrak{h} \otimes \mathfrak{h}, \mathfrak{h} \otimes \mathfrak{g}_{\alpha} \otimes \mathfrak{g}_{-\alpha}$ and $\mathfrak{g}_{\alpha} \otimes \mathfrak{g}_{\beta} \otimes \mathfrak{g}_{-\alpha-\beta}$ parts. With some short computation, the $\mathfrak{h} \otimes \mathfrak{h} \otimes \mathfrak{h}$ part can be seen to be

$$
\left.\operatorname{Alt}_{s}(d r)\right|_{\mathfrak{h} \otimes \mathfrak{h} \otimes \mathfrak{h}}=\sum_{i, j, k}\left(\frac{\partial D_{i j}}{x_{k}}+\frac{\partial D_{j k}}{x_{i}}+\frac{\partial D_{k i}}{x_{j}}\right) x_{i} \otimes x_{j} \otimes x_{k},
$$

and it vanishes if and only if $D=\sum_{i<j} D_{i j} d x_{i} \wedge d x_{j}$ is a closed 2-form.

To find the $\mathfrak{h} \otimes \mathfrak{g}_{\alpha} \otimes \mathfrak{g}_{-\alpha}$ part, we first look at $[[r, r]]$ :

$$
\begin{array}{r}
{\left[r^{12}, r^{13}\right]=\sum_{i, j, \beta} D_{i j} \phi_{\beta}\left[x_{i}, e_{\beta}\right] \otimes x_{j} \otimes e_{-\beta}+\sum_{k, l, \alpha} D_{k l} \phi_{\alpha}\left[e_{\alpha}, x_{k}\right] \otimes e_{-\alpha} \otimes x_{l}} \\
+\sum_{\alpha, \beta}(-1)^{|\alpha||\beta|} \phi_{\alpha} \phi_{\beta}\left[e_{\alpha}, e_{\beta}\right] \otimes e_{-\alpha} \otimes e_{-\beta}, \\
{\left[r^{12}, r^{23}\right]=\sum_{i, j, \beta} D_{i j} \phi_{\beta} x_{i} \otimes\left[x_{j}, e_{\beta}\right] \otimes e_{-\beta}+\sum_{k, l, \alpha} D_{k l} \phi_{\alpha} e_{\alpha} \otimes\left[e_{-\alpha}, x_{k}\right] \otimes x_{l}} \\
+\sum_{\alpha, \beta} \phi_{\alpha} \phi_{\beta} e_{\alpha} \otimes\left[e_{-\alpha}, e_{\beta}\right] \otimes e_{-\beta}, \\
{\left[r^{13}, r^{23}\right]=\sum_{i, j, \beta} D_{i j} \phi_{\beta} x_{i} \otimes e_{\beta} \otimes\left[x_{j}, e_{-\beta}\right]+\sum_{k, l, \alpha} D_{k l} \phi_{\alpha} e_{\alpha} \otimes x_{k} \otimes\left[e_{-\alpha}, x_{l}\right]} \\
+\sum_{\alpha, \beta}(-1)^{|\alpha||\beta|} \phi_{\alpha} \phi_{\beta} e_{\alpha} \otimes e_{\beta} \otimes\left[e_{-\alpha}, e_{-\beta}\right] .
\end{array}
$$

We can now easily see that the $\mathfrak{h} \otimes \mathfrak{g}_{\alpha} \otimes \mathfrak{g}_{-\alpha}$ part will be

$$
\begin{array}{r}
\left.\operatorname{Alt}_{s}(d r)\right|_{\mathfrak{h} \otimes \mathfrak{g}_{\alpha} \otimes \mathfrak{g}_{-\alpha}}+\sum_{\alpha, \beta}(-1)^{|\alpha||\beta|} \delta_{\alpha,-\beta} \phi_{\alpha} \phi_{\beta}\left[e_{\alpha}, e_{\beta}\right] \otimes e_{-\alpha} \otimes e_{-\beta} \\
+\sum_{i, j, \beta} D_{i j} \phi_{\beta} x_{i} \otimes\left[x_{j}, e_{\beta}\right] \otimes e_{-\beta}+\sum_{i, j, \beta} D_{i j} \phi_{\beta} x_{i} \otimes e_{\beta} \otimes\left[x_{j}, e_{-\beta}\right] .
\end{array}
$$


We note that the last two sums cancel out, because

$$
e_{\beta} \otimes\left[x_{j}, e_{-\beta}\right]=(-\beta)\left(x_{j}\right) e_{\beta} \otimes e_{-\beta}=-\left(\beta\left(x_{j}\right)\right) e_{\beta} \otimes e_{-\beta}=-\left[x_{j}, e_{\beta}\right] \otimes e_{\beta},
$$

and the first two can be reorganized and presented as

$$
\sum_{i, \alpha} \frac{\partial \phi_{\alpha}}{\partial x_{i}} x_{i} \otimes e_{\alpha} \otimes e_{-\alpha}+\sum_{\alpha} A_{\alpha} \phi_{\alpha} \phi_{\alpha} h_{\alpha} \otimes e_{\alpha} \otimes e_{-\alpha}
$$

which we can rewrite as

$$
\sum_{\alpha \in \Delta}\left(\sum_{i} \frac{\partial \phi_{\alpha}}{\partial x_{i}} x_{i}+A_{\alpha} \phi_{\alpha}^{2} h_{\alpha}\right) \otimes e_{\alpha} \otimes e_{-\alpha},
$$

where $h_{\alpha} \in \mathfrak{h}$ is defined by

$$
\left[e_{\alpha}, e_{-\alpha}\right]=\left(e_{\alpha}, e_{-\alpha}\right) h_{\alpha}=A_{-\alpha} h_{\alpha} .
$$

(Recall that both $\left\{x_{i}\right\}$ and $\left\{h_{\alpha} \mid \alpha \in \Gamma\right\}$ are bases for $\mathfrak{h}$ and equivalently linear coordinate systems for $\mathfrak{h}^{*}$.) For this term to vanish we must have, for all $\alpha \in \Delta$,

$$
\sum_{i} \frac{\partial \phi_{\alpha}}{\partial x_{i}} x_{i}+A_{\alpha} \phi_{\alpha}^{2} h_{\alpha}=0
$$

We can rewrite this as

$$
d \phi_{\alpha}+A_{\alpha} \phi_{\alpha}^{2} d h_{\alpha}=0
$$

If we define $\mu_{\alpha}$ by

$$
\mu_{\alpha}=\left\{\begin{array}{cl}
\sqrt{-1} & \text { if } \alpha \text { is an odd positive root } \\
1 & \text { otherwise }
\end{array}\right.
$$

then $\mu_{\alpha}^{2}=A_{\alpha}$, and the equation we need to solve is

$$
d \phi_{\alpha}+\mu_{\alpha}^{2} \phi_{\alpha}^{2} d h_{\alpha}=0 .
$$

We assume $\phi_{\alpha} \neq 0$ and let $u_{\alpha}=\mu_{\alpha} \phi_{\alpha}$. Separating variables to integrate we obtain

$$
\frac{1}{\mu_{\alpha}} \int \frac{-d u_{\alpha}}{u_{\alpha}^{2}}=\int d h_{\alpha} \Longrightarrow u_{\alpha}=\mu_{\alpha} \phi_{\alpha}=\frac{1}{\mu_{\alpha} h_{\alpha}+C}
$$

which gives us

$$
\phi_{\alpha}=\frac{A_{\alpha}}{h_{\alpha}-\nu_{\alpha}}
$$

for some $\nu_{\alpha} \in \mathbb{C}$. Here $h_{\alpha}$ is viewed as a linear function on $\mathfrak{h}^{*}$ via $h_{\alpha}(\lambda)=(\alpha, \lambda)$.

Finally we look at the $\mathfrak{g}_{\alpha} \otimes \mathfrak{g}_{\beta} \otimes \mathfrak{g}_{-\alpha-\beta}$ part of Equation (2.1). There is no contribution from the dynamical part; the only terms we need to look at are

$$
\begin{array}{r}
\sum_{\alpha, \beta}(-1)^{|\alpha||\beta|} \phi_{\alpha} \phi_{\beta}\left[e_{\alpha}, e_{\beta}\right] \otimes e_{-\alpha} \otimes e_{-\beta}+\sum_{\alpha, \beta} \phi_{\alpha} \phi_{\beta} e_{\alpha} \otimes\left[e_{-\alpha}, e_{\beta}\right] \otimes e_{-\beta} \\
+\sum_{\alpha, \beta}(-1)^{|\alpha||\beta|} \phi_{\alpha} \phi_{\beta} e_{\alpha} \otimes e_{\beta} \otimes\left[e_{-\alpha}, e_{-\beta}\right] .
\end{array}
$$

Denote by $C$ the matrix of coefficients determined by

$$
\left.\left[e_{\alpha}, e_{\beta}\right]=C_{\alpha, \beta}^{\gamma} e_{\gamma} . \quad \text { (Note that } C_{\alpha, \beta}^{\gamma}=0 \text { if } \gamma \neq \alpha+\beta .\right)
$$


Then after some simplifications, we can rewrite the terms we are interested in as

$$
\begin{array}{r}
\sum_{\alpha, \beta}(-1)^{|\alpha||\beta|} \phi_{\alpha} \phi_{\beta} C_{\alpha, \beta}^{\alpha+\beta} e_{\alpha+\beta} \otimes e_{-\alpha} \otimes e_{-\beta}+\sum_{\alpha, \beta} \phi_{\alpha} \phi_{\beta} C_{-\alpha, \beta}^{-\alpha+\beta} e_{\alpha} \otimes e_{-\alpha+\beta} \otimes e_{-\beta} \\
+\sum_{\alpha, \beta}(-1)^{|\alpha||\beta|} \phi_{\alpha} \phi_{\beta} C_{-\alpha,-\beta}^{-\alpha-\beta} e_{\alpha} \otimes e_{\beta} \otimes e_{-\alpha-\beta} .
\end{array}
$$

We want the coefficient in front of the term $e_{\alpha} \otimes e_{\beta} \otimes e_{-\alpha-\beta}$ to vanish

$$
\begin{aligned}
(-1)^{|\beta||\alpha+\beta|} \phi_{-\beta} \phi_{\alpha+\beta} C_{-\beta, \alpha+\beta}^{\alpha}+\phi_{\alpha} \phi_{\alpha+\beta} C_{-\alpha, \alpha+\beta}^{\beta} & \\
+(-1)^{|\alpha||\beta|} \phi_{\alpha} \phi_{\beta} C_{-\alpha,-\beta}^{-\alpha-\beta} & =0
\end{aligned}
$$

or equivalently

$$
\begin{array}{r}
-(-1)^{|\alpha||\beta|} \phi_{\beta} \phi_{\alpha+\beta} C_{-\beta, \alpha+\beta}^{\alpha}+\phi_{\alpha} \phi_{\alpha+\beta} C_{-\alpha, \alpha+\beta}^{\beta} \\
+(-1)^{|\alpha||\beta|} \phi_{\alpha} \phi_{\beta} C_{-\alpha,-\beta}^{-\alpha-\beta}=0 .
\end{array}
$$

Using the invariance and supersymmetry of the Killing form, we obtain

$$
\begin{aligned}
C_{-\beta, \alpha+\beta}^{\alpha} & =\left(\left[e_{-\beta}, e_{\alpha+\beta}\right], e_{\alpha}^{*}\right)=\left(\left[e_{-\beta}, e_{\alpha+\beta}\right], A_{-\alpha} e_{-\alpha}\right) \\
& =A_{-\alpha}\left(\left[e_{-\beta}, e_{\alpha+\beta}\right], e_{-\alpha}\right), \\
C_{-\alpha, \alpha+\beta}^{\beta} & =\left(\left[e_{-\alpha}, e_{\alpha+\beta}\right], e_{\beta}^{*}\right)=\left(\left[e_{-\alpha}, e_{\alpha+\beta}\right], A_{-\beta} e_{-\beta}\right) \\
& =-(-1)^{|\alpha|}(-1)^{|\beta|}(-1)^{|\alpha||\beta|} A_{-\beta}\left(\left[e_{-\beta}, e_{\alpha+\beta}\right], e_{-\alpha}\right), \\
C_{-\alpha,-\beta}^{-\alpha-\beta} & =\left(\left[e_{-\alpha}, e_{-\beta}\right], e_{-\alpha-\beta}^{*}\right)=\left(\left[e_{-\alpha}, e_{-\beta}\right], A_{\alpha+\beta} e_{\alpha+\beta}\right) \\
& =(-1)^{|\alpha|} A_{\alpha+\beta}\left(\left[e_{-\beta}, e_{\alpha+\beta}\right], e_{-\alpha}\right) .
\end{aligned}
$$

Plugging these values for $C$ into the equation for the coefficient of $e_{\alpha} \otimes e_{\beta} \otimes e_{-\alpha-\beta}$ and simplifying we get

$$
0=-(-1)^{|\beta|} \phi_{\beta} \phi_{\alpha+\beta} A_{-\alpha}-(-1)^{|\alpha|} \phi_{\alpha} \phi_{\alpha+\beta} A_{-\beta}+(-1)^{|\alpha|+|\beta|} \phi_{\alpha} \phi_{\beta} A_{\alpha+\beta},
$$

which can be rewritten as

$$
A_{\alpha+\beta} \phi_{\alpha} \phi_{\beta}=\left(A_{\alpha} \phi_{\beta}+A_{\beta} \phi_{\alpha}\right) \phi_{\alpha+\beta},
$$

where we use $A_{\alpha}=(-1)^{|\alpha|} A_{-\alpha}$ and $\left(A_{\alpha}\right)^{2}=1$.

Define the set $X=\left\{\alpha \in \Delta \mid \phi_{\alpha} \neq 0\right\}$. $X$ is closed under addition and changing signs. In other words, if $\alpha, \beta \in X$, then so are $\alpha+\beta$ and $-\alpha$. These follow directly from Equation (3.2) and the unitarity property (i.e. $r=-T_{s}(r)$ ).

Next assume $\alpha$ and $\beta$ are positive and in $X$. Then Equation (3.2) becomes

$$
\begin{aligned}
(-1)^{|\alpha|+|\beta|} \phi_{\alpha} \phi_{\beta} & =\left((-1)^{|\alpha|} \phi_{\beta}+(-1)^{|\beta|} \phi_{\alpha}\right) \phi_{\alpha+\beta}, \\
(-1)^{|\alpha|+|\beta|}\left(\frac{(-1)^{|\alpha|}}{h_{\alpha}-\nu_{\alpha}}\right)\left(\frac{(-1)^{|\beta|}}{h_{\beta}-\nu_{\beta}}\right) & =\left(\frac{(-1)^{|\alpha+\beta|}}{h_{\beta}-\nu_{\beta}}+\frac{(-1)^{|\alpha+\beta|}}{h_{\alpha}-\nu_{\alpha}}\right) \frac{(-1)^{|\alpha+\beta|}}{h_{\alpha+\beta}-\nu_{\alpha+\beta}} \\
\left(\frac{1}{h_{\alpha}-\nu_{\alpha}}\right)\left(\frac{1}{h_{\beta}-\nu_{\beta}}\right) & =\left(\frac{1}{h_{\beta}-\nu_{\beta}}+\frac{1}{h_{\alpha}-\nu_{\alpha}}\right) \frac{1}{h_{\alpha+\beta}-\nu_{\alpha+\beta}} .
\end{aligned}
$$

Since $h_{\alpha+\beta}=\mathfrak{h}_{\alpha}+h_{\beta}$ and $\phi_{-\alpha}=-(-1)^{|\alpha|} \phi_{\alpha}$, we must have

$$
\nu_{\alpha+\beta}=\nu_{\alpha}+\nu_{\beta} \quad \text { and } \quad \frac{A_{-\alpha}}{h_{-\alpha}-\nu_{-\alpha}}=-(-1)^{|\alpha|} \frac{A_{\alpha}}{h_{\alpha}-\nu_{\alpha}},
$$


and the latter implies that $\nu_{-\alpha}=-\nu_{\alpha}$. Therefore there is some $\nu \in \mathfrak{h}^{*}$ such that $\nu_{\alpha}=(\alpha, \nu)$ for all $\alpha \in X$. This completes the proof of Theorem 2.2.

\section{Proof of Theorem 2.3}

We begin by fixing a basis for the non-Cartan part of $\mathfrak{g}$ as in Section 3 . We will also need $A_{\alpha}$ as defined there by Equation (3.1).

Proof. Let $r: \mathfrak{h}^{*} \rightarrow \mathfrak{g} \otimes \mathfrak{g}$ be a meromorphic map, $\Omega \in \mathfrak{g} \otimes \mathfrak{g}$ the Casimir element, and $\epsilon$ a non-zero complex number. Introduce a second meromorphic function $s: \mathfrak{h}^{*} \rightarrow \mathfrak{g} \otimes \mathfrak{g}$ by

$$
s(\lambda)=r(\lambda)-\frac{\epsilon}{2} \Omega \quad \text { for all } \lambda \in \mathfrak{h}^{*} .
$$

We will need a technical lemma, which is a natural superization of Lemma 3.9 of [6. Its proof consists mainly of a direct calculation, and even though the computations are significantly more involved in the super case, we will not include them here.

Lemma 4.1. The map $r$ is a super dynamical $r$-matrix with zero weight and coupling constant $\epsilon$ if and only if $s$ satisfies the zero weight condition

$$
[h \otimes 1+1 \otimes h, s(\lambda)]=0 \text { for all } h \in \mathfrak{h}, \lambda \in \mathfrak{h}^{*},
$$

the unitarity condition

$$
s(\lambda)+T_{s}(s)(\lambda)=0,
$$

and the following modified version of the dynamical Yang-Baxter equation:

$$
\operatorname{Alt}_{s}(d s)+[[s, s]]+\frac{\epsilon^{2}}{4}[[\Omega, \Omega]]=0 .
$$

This lemma shows that in order to study zero-weight super dynamical $r$-matrices with a non-zero coupling constant, it suffices to solve Equation (4.1). The zero weight condition and the unitarity condition on $s$ imply that $s$ has to be of the form

$$
s(\lambda)=\sum_{i, j} D_{i j}(\lambda) h_{i} \otimes h_{j}+\sum_{\alpha \in \Delta} \phi_{\alpha} e_{\alpha} \otimes e_{-\alpha}
$$

where $\left\{h_{i}\right\}$ is a basis for $\mathfrak{h}$, and $D_{i j}, \phi_{\alpha}$ are suitable scalar meromorphic functions such that

$$
D_{i j}(\lambda)=-D_{j i}(\lambda) \quad \text { and } \quad \phi_{-\alpha}=-(-1)^{|\alpha|} \phi_{\alpha} .
$$

One can easily check that there can be no terms mixing the Cartan part with the non-Cartan part, and the conditions on the $D_{i j}$ and the $\phi_{\alpha}$ follow from unitarity.

Equation (4.1) is an equation in $\mathfrak{g} \otimes \mathfrak{g} \otimes \mathfrak{g}$. The non-Casimir components are easily seen to be skew-symmetric with respect to signed permutations of factors, following arguments from Section 3 . The Casimir part is also skew-symmetric with respect to signed permutations of factors, as can be seen by a straightforward computation. Because of this symmetry, it is sufficient to look only at the $\mathfrak{h} \otimes \mathfrak{h} \otimes \mathfrak{h}, \mathfrak{h} \otimes \mathfrak{g}_{\alpha} \otimes \mathfrak{g}_{-\alpha}$ and $\mathfrak{g}_{\alpha} \otimes \mathfrak{g}_{\beta} \otimes \mathfrak{g}_{-\alpha-\beta}$ components in order to solve Equation (4.1).

The $\mathfrak{h} \otimes \mathfrak{h} \otimes \mathfrak{h}$ part comes only from the dynamical part (in other words, from $\left.\operatorname{Alt}_{s}(d s)\right)$ and yields

$$
\frac{\partial D_{j k}}{\partial x_{i}}+\frac{\partial D_{k i}}{\partial x_{j}}+\frac{\partial D_{i j}}{\partial x_{k}}=0
$$

which implies that $D=\sum_{i<j} D_{i j} d x_{i} \wedge d x_{j}$ is a closed differential 2-form. 
The $\mathfrak{h} \otimes \mathfrak{g}_{\alpha} \otimes \mathfrak{g}_{-\alpha}$ part consists of two components. The part that comes from the non-Casimir part is the same as the whole of the $\mathfrak{h} \otimes \mathfrak{g}_{\alpha} \otimes \mathfrak{g}-\alpha$ part of the dynamical Yang-Baxter equation for a zero-weight super dynamical $r$-matrix with zero coupling constant (see Section 3). To this we need to add the Casimir component, i.e. the terms that come from $\frac{\epsilon^{2}}{4}[[\Omega, \Omega]]$, which after some simplifications, become

$$
\left.[[\Omega, \Omega]]\right|_{\mathfrak{h} \otimes \mathfrak{g}_{\alpha} \otimes \mathfrak{g}_{-\alpha}}=-\sum_{\alpha} A_{\alpha} h_{\alpha} \otimes e_{\alpha} \otimes e_{-\alpha},
$$

and so the $\mathfrak{h} \otimes \mathfrak{g}_{\alpha} \otimes \mathfrak{g}_{-\alpha}$ part of Equation (4.1) is

$$
\sum_{i, \alpha} \frac{\partial \phi_{\alpha}}{\partial x_{i}} x_{i} \otimes e_{\alpha} \otimes e_{-\alpha}+\sum_{\alpha} A_{\alpha} \phi_{\alpha}^{2} h_{\alpha} \otimes e_{\alpha} \otimes e_{-\alpha}-\frac{\epsilon^{2}}{4} \sum_{\alpha} A_{\alpha} h_{\alpha} \otimes e_{\alpha} \otimes e_{-\alpha}
$$

which we can rewrite as

$$
\sum_{\alpha \in \Delta}\left(\sum_{i} \frac{\partial \phi_{\alpha}}{\partial x_{i}} x_{i}+A_{\alpha}\left(\phi_{\alpha}^{2}-\frac{\epsilon^{2}}{4}\right) h_{\alpha}\right) \otimes e_{\alpha} \otimes e_{-\alpha}
$$

For this term to vanish we must have, for all $\alpha \in \Delta$

$$
\sum_{i} \frac{\partial \phi_{\alpha}}{\partial x_{i}} x_{i}+A_{\alpha}\left(\phi_{\alpha}^{2}-\frac{\epsilon^{2}}{4}\right) h_{\alpha}=0
$$

We can rewrite this as

$$
d \phi_{\alpha}+A_{\alpha}\left(\phi_{\alpha}^{2}-\frac{\epsilon^{2}}{4}\right) d h_{\alpha}=0
$$

If $\mu_{\alpha}$ is defined as in Section 3. the equation we need to solve is

$$
d \phi_{\alpha}+\mu_{\alpha}^{2}\left(\phi_{\alpha}^{2}-\frac{\epsilon^{2}}{4}\right) d h_{\alpha}=0
$$

We assume $\phi_{\alpha}^{2} \neq \frac{\epsilon^{2}}{4}$ and let $u_{\alpha}=\mu_{\alpha} \phi_{\alpha}$. Separating variables to integrate we obtain

$$
\frac{1}{\mu_{\alpha}} \int \frac{d u_{\alpha}}{\epsilon_{\alpha}^{2}-u_{\alpha}^{2}}=\int d h_{\alpha} \Longrightarrow u_{\alpha}=\mu_{\alpha} \phi_{\alpha}=\epsilon_{\alpha} \operatorname{coth}\left(\epsilon_{\alpha} \mu_{\alpha} h_{\alpha}+C\right),
$$

where $\epsilon_{\alpha}=\frac{\mu_{\alpha} \epsilon}{2}$. We get

$$
\phi_{\alpha}=\frac{\epsilon}{2} \operatorname{coth}\left(\frac{A_{\alpha} \epsilon}{2}\left(h_{\alpha}-\nu_{\alpha}\right)\right)
$$

for some $\nu_{\alpha} \in \mathbb{C}$. Here $h_{\alpha}$ is viewed as a linear function on $\mathfrak{h}^{*}$ via $h_{\alpha}(\lambda)=(\alpha, \lambda)$.

Finally we look at the $\mathfrak{g}_{\alpha} \otimes \mathfrak{g}_{\beta} \otimes \mathfrak{g}_{-\alpha-\beta}$ part of Equation (4.1). There is no contribution from the dynamical part. The terms from $[[s, s]]$ are

$$
\begin{array}{r}
\sum_{\alpha, \beta}(-1)^{|\alpha||\beta|} \phi_{\alpha} \phi_{\beta}\left[e_{\alpha}, e_{\beta}\right] \otimes e_{-\alpha} \otimes e_{-\beta}+\sum_{\alpha, \beta} \phi_{\alpha} \phi_{\beta} e_{\alpha} \otimes\left[e_{-\alpha}, e_{\beta}\right] \otimes e_{-\beta} \\
+\sum_{\alpha, \beta}(-1)^{|\alpha||\beta|} \phi_{\alpha} \phi_{\beta} e_{\alpha} \otimes e_{\beta} \otimes\left[e_{-\alpha}, e_{-\beta}\right],
\end{array}
$$

and the coefficient for the $e_{\alpha} \otimes e_{\beta} \otimes e_{-\alpha-\beta}$ term coming from this component is

$$
(-1)^{|\beta||\alpha+\beta|} \phi_{-\beta} \phi_{\alpha+\beta} C_{-\beta, \alpha+\beta}^{\alpha}+\phi_{\alpha} \phi_{\alpha+\beta} C_{-\alpha, \alpha+\beta}^{\beta}+(-1)^{|\alpha||\beta|} \phi_{\alpha} \phi_{\beta} C_{-\alpha,-\beta}^{-\alpha-\beta} .
$$

Further simplification yields

$$
-\phi_{\beta} \phi_{\alpha+\beta} \frac{A_{\beta}}{C_{\alpha, \beta}^{\alpha+\beta}}\left(h_{\alpha}, h_{\beta}\right)-\phi_{\alpha} \phi_{\alpha+\beta} \frac{A_{\alpha}}{C_{\alpha, \beta}^{\alpha+\beta}}\left(h_{\alpha}, h_{\beta}\right)+\phi_{\alpha} \phi_{\beta} \frac{A_{\alpha+\beta} A_{\alpha} A_{\beta}}{C_{\alpha, \beta}^{\alpha+\beta}}\left(h_{\alpha}, h_{\beta}\right) .
$$


The Casimir component is

$$
\begin{array}{r}
\frac{\epsilon^{2}}{4}\left(\sum_{\alpha, \beta}(-1)^{|\alpha||\beta|} A_{\alpha} A_{\beta}\left[e_{\alpha}, e_{\beta}\right] \otimes e_{-\alpha} \otimes e_{-\beta}+\sum_{\alpha, \beta} A_{\alpha} A_{\beta} e_{\alpha} \otimes\left[e_{-\alpha}, e_{\beta}\right] \otimes e_{-\beta}\right. \\
\left.+\sum_{\alpha, \beta}(-1)^{|\alpha||\beta|} A_{\alpha} A_{\beta} e_{\alpha} \otimes e_{\beta} \otimes\left[e_{-\alpha}, e_{-\beta}\right]\right),
\end{array}
$$

and the coefficient in front of the $e_{\alpha} \otimes e_{\beta} \otimes e_{-\alpha-\beta}$ term coming from this component is

$$
\begin{aligned}
\frac{\epsilon^{2}}{4}\left((-1)^{|\beta||\alpha+\beta|} A_{-\beta} A_{\alpha+\beta} C_{-\beta, \alpha+\beta}^{\alpha}\right. & +A_{\alpha} A_{\alpha+\beta} C_{-\alpha, \alpha+\beta}^{\beta} \\
& \left.+(-1)^{|\alpha||\beta|} A_{\alpha} A_{\beta} C_{-\alpha,-\beta}^{-\alpha-\beta}\right),
\end{aligned}
$$

which further simplifies to

$$
\frac{\epsilon^{2}}{4}\left(\frac{A_{\alpha+\beta}}{C_{\alpha, \beta}^{\alpha+\beta}}\left(h_{\alpha}, h_{\beta}\right)\right)
$$

Therefore the coefficient of the $e_{\alpha} \otimes e_{\beta} \otimes e_{-\alpha-\beta}$ term vanishes if and only if

$$
\phi_{\beta} \phi_{\alpha+\beta} A_{\beta}+\phi_{\alpha} \phi_{\alpha+\beta} A_{\alpha}=\phi_{\alpha} \phi_{\beta} A_{\alpha+\beta} A_{\alpha} A_{\beta}+\frac{\epsilon^{2}}{4} A_{\alpha+\beta},
$$

or equivalently

$$
A_{\alpha+\beta} \phi_{\alpha} \phi_{\beta}+\frac{\epsilon^{2}}{4} A_{\alpha+\beta} A_{\alpha} A_{\beta}=\phi_{\alpha+\beta}\left(A_{\alpha} \phi_{\beta}+A_{\beta} \phi_{\alpha}\right),
$$

where we use $A_{\alpha}^{2}=1$.

Let $X=\left\{\alpha \in \Delta \mid \phi_{\alpha}^{2} \neq \frac{\epsilon^{2}}{4}\right\}$. Then clearly $X$ is closed under changing signs because $s$ satisfies the unitarity condition. Equation (4.3) implies that $X$ is also closed under addition. If $\alpha, \beta \in X$ are two positive roots, then

$$
\begin{aligned}
\phi_{\alpha} & =\frac{\epsilon}{2} \operatorname{coth}\left(\frac{A_{\alpha} \epsilon}{2}\left(h_{\alpha}-\nu_{\alpha}\right)\right), \\
\phi_{\beta} & =\frac{\epsilon}{2} \operatorname{coth}\left(\frac{A_{\beta} \epsilon}{2}\left(h_{\beta}-\nu_{\beta}\right)\right),
\end{aligned}
$$

and Equation (4.3) implies that

$$
\phi_{\alpha+\beta}=\frac{\epsilon}{2} A_{\alpha+\beta} \operatorname{coth}\left(\frac{\epsilon}{2}\left(h_{\alpha}-\nu_{\alpha}\right)+\frac{\epsilon}{2}\left(h_{\beta}-\nu_{\beta}\right)\right)
$$

is not constant, so we must have

$$
\phi_{\alpha+\beta}=\frac{\epsilon}{2} \operatorname{coth}\left(\frac{A_{\alpha+\beta} \epsilon}{2}\left(h_{\alpha+\beta}-\nu_{\alpha+\beta}\right)\right) .
$$

Since $\operatorname{coth}(-x)=-\operatorname{coth}(x)$, this also implies that

$$
\nu_{\alpha+\beta}=\nu_{\alpha}+\nu_{\beta}
$$

Using $\phi_{-\alpha}=-(-1)^{|\alpha|} \phi_{\alpha}$ we also see that $\nu_{-\alpha}=-\nu_{\alpha}$. Hence, there is some $\nu \in \mathfrak{h}^{*}$ with $\nu_{\alpha}=(\alpha, \nu)$ for all $\alpha \in X$, and the proof of Theorem 2.3 is thus complete. 


\section{EXAMPLES OF SUPER DYNAMICAL $r$-MATRICES}

Example 5.1. Let $\mathfrak{g}$ be a simple Lie superalgebra with nondegenerate Killing form. For any choice of a triangular decomposition of $\mathfrak{g}$, the constant $r$-matrix

$$
r=\frac{\epsilon}{2} \sum_{i}\left(x_{i} \otimes x_{i}^{*}\right)+\epsilon \sum_{\alpha \in \Delta^{+}}\left(e_{-\alpha} \otimes e_{\alpha}\right)
$$

and its super twist

$$
T_{s}(r)=\frac{\epsilon}{2} \sum_{i}\left(x_{i} \otimes x_{i}^{*}\right)+\epsilon \sum_{\alpha \in \Delta^{+}}\left((-1)^{|\alpha|} e_{\alpha} \otimes e_{-\alpha}\right)
$$

are both zero-weight super dynamical $r$-matrices with coupling constant $\epsilon$. They correspond to $X=\emptyset$ and $D \equiv 0$ in Theorem 2.3. We note that these $r$-matrices are in fact solutions to the classical Yang-Baxter equation. In fact, $r$ can clearly be constructed by the main construction theorem of [8] with the trivial admissible triple $\left(\Gamma_{1}, \Gamma_{2}, \tau\right)$ where $\Gamma_{i}=\emptyset$ and $\tau=0 \unrhd$

Remark 5.2. The only solution of the classical Yang-Baxter equation constructible by the main construction theorem of [8] that we can also construct by Theorem 2.3 is $r$ given above. To reach any $r$-matrix that involves a non-trivial admissible triple, we would need to drop the zero weight condition.

Example 5.3. For a non-constant dynamical example, consider the super dynamical $r$-matrix constructed by Theorem 2.3 using $X=\Delta, D \equiv 0$ and $\nu=0$ :

$$
r(\lambda)=\frac{\epsilon}{2} \Omega+\sum_{\alpha \in \Delta} \frac{\epsilon}{2} \operatorname{coth}\left(\frac{\epsilon}{2}(\alpha, \lambda)\right)(-1)^{|\alpha|}\left(e_{\alpha}, e_{-\alpha}\right)\left(e_{\alpha} \otimes e_{-\alpha}\right) .
$$

For a given triangular decomposition this can be rewritten as

$$
r(\lambda)=\frac{\epsilon}{2} \Omega+\sum_{\alpha \in \Delta^{+}} \frac{\epsilon}{2} \operatorname{coth}\left(\frac{\epsilon}{2}(\alpha, \lambda)\right)\left((-1)^{|\alpha|} e_{\alpha} \otimes e_{-\alpha}-e_{-\alpha} \otimes e_{\alpha}\right) .
$$

For this triangular decomposition, if we take the limit as $\lambda \rightarrow \infty$ while always staying positive, then we see that

$$
r(\lambda) \longrightarrow \frac{\epsilon}{2} \Omega+\sum_{\alpha \in \Delta^{+}} \frac{\epsilon}{2}\left((-1)^{|\alpha|} e_{\alpha} \otimes e_{-\alpha}-e_{-\alpha} \otimes e_{\alpha}\right),
$$

which is exactly the $r$-matrix $T_{s}(r)$ of the previous example. If we take a similar limit as $|\lambda| \rightarrow \infty$ while always staying negative, we see that

$$
r(\lambda) \longrightarrow \frac{\epsilon}{2} \Omega+\sum_{\alpha \in \Delta^{+}} \frac{\epsilon}{2}\left(e_{-\alpha} \otimes e_{\alpha}-(-1)^{|\alpha|} e_{\alpha} \otimes e_{-\alpha}\right),
$$

which is exactly the $r$-matrix $r$ of the previous example. Hence we can conclude that this particular super dynamical $r$-matrix extrapolates different solutions of the classical Yang-Baxter equation associated to the trivial admissible triple, labeled by different triangular decompositions.

\footnotetext{
${ }^{2}$ Here we use $x_{i}^{*} \in \mathfrak{h}$, the elements of the dual basis of the basis $\left\{x_{i}\right\}$ for $\mathfrak{h}$, because it may not always be possible to choose an orthonormal basis for $\mathfrak{h}$ in the super case.
} 


\section{REFERENCES}

1. Belavin, A. A., Drinfeld, V. G.; "Solutions of the Classical Yang-Baxter Equation and Simple Lie Algebras", Funct. Anal. Appl. 16 (1982), pp.159-180. MR0674005 (84e:81034)

2. Belavin, A. A., Drinfeld, V. G.; "Triangle Equation and Simple Lie Algebras", Soviet Scientific Reviews Sect. C 4 (1984), pp.93-165. MR0768939 (87h:58078)

3. Etingof, P.; "On the Dynamical Yang-Baxter Equation", Proceedings of the International Congress of Mathematicians, Vol. II (Beijing, 2002), Higher Ed. Press, 2002, pp.555-570. MR 1957065 (2004k:17025)

4. Etingof, P., Schedler, T., Schiffmann, O.; "Explicit Quantization of Dynamical r-matrices for Finite Dimensional Semisimple Lie Algebras", J. Amer. Math. Soc. 13 (2000), no. 3, pp.595-609. MR.1758755 (2001j:17024)

5. Etingof, P., Schiffmann, O.; Lectures on Quantum Groups, International Press, 1998. MR.1698405 (2000e:17016)

6. Etingof, P., Varchenko, A.; "Geometry and Classification of Solutions of the Classical Dynamical Yang-Baxter Equation", Comm. Math. Phys. 192 (1998), no. 1, pp.77-120. MR1612160 (99e:32032)

7. Geer, N.; "Etingof-Kazhdan Quantization of Lie Superbialgebras"; arXiv:math.QA/0409563

8. Karaali, G.; "Constructing r-matrices on Simple Lie Superalgebras", J. Algebra 282 (2004), no.1, pp.83-102. MR2095573(2005h:17017)

9. Karaali, G.; "A New Lie Bialgebra Structure on sl $(2,1)$ ", Contemp. Math. (to appear).

10. Schiffmann, O.; "On Classification of Dynamical r-matrices", Math. Res. Lett. 5 (1998), pp.13-30. MR1618367 (99j:17026)

Department of Mathematics, University of California, Santa Barbara, California 93106

E-mail address: gizem@math.ucsb.edu 